\title{
Perfection du matin de Sharon Butala, ou comment élargir la langue minoritaire
}

\author{
Nicole Côté \\ University of Regina
}

\section{Introduction : la traduction comme cartographie identitaire}

J'ai accepté de traduire ${ }^{1}$ The Perfection of the Morning de Sharon Butala, une méditation personnelle sur la terre et sur la vie presque disparue des ranchers dans la région désertique et aride du sud de la Saskatchewan, parce qu'un certain nombre de Fransaskois considéraient comme importante la traduction de ce livre en français. En effet, ces Fransaskois appréciaient l'Ouest que Sharon Butala avait esquissé et voulaient une représentation en français de ce paysage qu'on associe exclusivement au Canada anglais et aux États-Unis, puisque les grandes fermes de monocultures n'existent pas dans le monde francophone. Traduire cette réalité, $c^{\prime}$ était donner des mots à une communauté francophone qui a vécu sur cette terre, et où ses enfants vivront peut-être.

Je voudrais donc - une fois présentées les particularités de The Perfection of the Morning - dans un premier temps, montrer comment la traduction d'une réalité majoritairement anglophone peut permettre aux francophones minoritaires de se l'approprier; j'espère également que cette traduction pourra contribuer à élargir leur langue tout en rendant compte de leur monde hybride, métissé ${ }^{2}$ Je me suis par ailleurs interrogée sur les motifs qui ont poussé des Fransaskois à demander aux Éditions de la nouvelle plume la traduction de ce texte, et j'aimerais par conséquent, dans un deuxième temps, discuter certains

\footnotetext{
${ }^{1}$ Anton Iorga a formé équipe avec moi et a traduit certains chapitres.

${ }^{2}$ Ayant justifié mon entreprise, je voudrais mettre un bémol en mentionnant brièvement mes réticences. D'abord, cette œuvre particulière n'étant pas de la fiction, j'avais des réserves quant à la traduction d'idées avec lesquelles je pouvais ne pas être d'accord - et je les ai toujours. Par ailleurs, un autre problème, celui-là d'envergure, se posait. Comment une Québécoise et, $a$ fortiori, une citadine, connaîtrait-elle quoi que ce soit sur la vie de ranch, dans le paysage très particulier du Sud de la Saskatchewan - un paysage qui doit plus au Montana et au Nevada dans sa formation géologique qu'à aucun autre paysage canadien ? C'est grâce aux séances de travail avec Butala, principalement durant notre séjour à Banff, que je suis venue à bout de la plupart des difficultés posées par l'interpétation du monde physique décrit dans Perfection du matin. Termium et Parcs Canada m'ont été d'une grande aide, de même que mes collègues traductrices du département de français, Joanne Bonneville, son mari Lionel Bonneville, ainsi que Brigitte Haguès.
} 
thèmes ou caractéristiques de l'œuvre qui semblent agir comme miroir de la condition minoritaire.

\section{Radiographie de l'œuvre : thèmes et frontières génériques}

Perfection $d u$ matin est une très belle méditation sur la terre, sur le mode de vie presque éteint des ranchers, mais également sur le voyage émotif et spirituel d'une femme de la ville qui doit vivre dans un milieu rural incroyablement isolé. En outre, et contrairement à Brokeback Mountain - la nouvelle d'Annie Proulx dont Ang Lee a tiré un film fétiche - le point de vue de la narration est ici celui d'une femme, un angle doublement rare dans la représentation du Far-Ouest en ce qu'il est féminin et en français. La traduction garde donc la représentation d'une expérience typiquement anglo-canadienne tout en prolongeant la vie de l'œuvre dans un autre contexte linguistique et culturel.

L'œuvre The Perfection of the Morning intéresse aussi en ce qu'elle brouille les frontières génériques, bien qu'elle discute de la nature et d'un mode de vie en voie de disparition, des thèmes que l'on associe volontiers avec un genre traditionnel - de telle manière que sa forme hybride reflète clairement l'ère dans laquelle elle a été écrite. Ce sont les mémoires d'une jeune femme de milieu urbain et universitaire qui s'aventure dans une région reculée de la province, désertique et sauvage. Quoique Butala commence ses mémoires comme la chronique d'un peuple en voie de disparition - les ranchers, qui accomplissent à peu près toutes leurs tâches à dos de cheval -, elle inscrit dans cette chronique au quotidien les confessions d'une jeune artiste en herbe - Portrait de l'artiste en jeune cow-girl ${ }^{3}$, pourrait-on dire - qui lutte farouchement pour trouver sa place dans une communauté d'un conservatisme outrancier, où les femmes doivent accomplir une diversité remarquable de tâches tout en ayant un statut quasiinvisible. On voit donc comment une minorisation rejoint et reflète l'autre. En raison des expériences particulières qui ont motivé le discours de Butala jusquelà, son récit offre des représentations opposées - d'un côté, le monde sensoriel et concret des ranchers dans une nature restée presque intacte, et de l'autre, celle d'un monde intérieur aussi vaste que les plaines. Déjà le premier chapitre semble contenir en germe ces deux pôles: bien qu'il constitue en grande partie une

\footnotetext{
${ }^{3}$ Puisqu'il s'agit d'un récit de la formation d'un artiste, mon sous-titre imaginé veut attirer l'attention sur le type d'écrit - une sorte de bildungsroman sur la naissance de l'écrivain -, mais en mettant l'accent sur deux aspects insolites : ces mémoires sont écrits sur un ranch, et de surcroît, par une femme. Mon sous-titre humoristique s'inscrit dans la généalogie d'écrivains comme Dylan Thomas, qui, avec son Portrait of the Artist as a Young Dog, parodie James Joyce et son célèbre Portrait of the Artist as a Young Man.
} 
description factuelle de la vie quotidienne au ranch Butala, le chapitre entier garde une aura de poésie, comme le lyrisme retenu de la description du travail des cowboys au diapason de leur environnement. Le même chapitre contient également des descriptions de rêves qui semblent agir comme des guides vers le chemin inconnu et parfois terrifiant entrepris par l'auteure.

Butala explore donc dans son nouveau milieu sa lutte corps-à-corps avec la solitude et la dépression, prémisses de la profonde crise identitaire qui s'ensuivrait et qui permettrait la naissance d'un être nouveau, l'écrivaine. Cette période $d$ 'introspection permet à Butala d'explorer un registre d'émotions très étendu et particulièrement fin. Le chapitre intitulé "Anomie » est peut-être le plus purement introspectif, et est caractéristique de ce point de non-retour où l'évocation lyrique des promenades à cheval et à pied dans une nature resplendissante cède à une promenade à l'intérieur de soi. La très honnête description qu'a faite Butala du désespoir résultant du changement de contexte et de culture, sa brave tentative d'en faire sens en l'ordonnant en récit, constituent selon moi un document qui permettra peut-être également aux femmes franco-canadiennes, en particulier celles des milieux ruraux, de donner des mots dans leur langue à l'obscur désespoir d'être femme et minoritaire dans un pays où l'on considère généralement que la lutte pour le français est aussi gagnée que celle des droits des femmes parce qu'en théorie des lois les protègent. Il n'est en ce sens pas surprenant qu'à l'arrière-plan de ce récit d'apprentissage se remarque le leitmotif des Premières Nations, dont la présence dans la région où se situe le ranch Butala pourrait être considérée comme une mise en abîme de leur disparition sur le territoire canadien, comme un emblème de toutes les cultures ou groupes menacées.

Si la forme même de The Perfection of the Morning est composite au point où, par la variété du matériel abordé, elle pourrait être comparée à celle d'un almanach, on peut imaginer qu'il en est de même pour le vocabulaire. Parce que le genre dans lequel Butala écrit est toujours mixte, qu'il soit fiction ou autobiographie, l'éventail de ce vocabulaire s'élargit pour tenir compte des deux principaux registres - référentiel et poétique - qui y cohabitent. Même après avoir fait une rapide lecture de l'œuvre, je n'étais pas préparée à la variété des terminologies intégrées au discours de Butala : terminologie du ranch et de la ferme de monoculture; terminologie géographique, géologique et météorologique, botanique, archéologique et anthropologique, pour ne nommer que quelques-uns des domaines liés à cet espace de la Plaine du sud-ouest de la Saskatchewan. 
Le style de The Perfection of the Morning n'est pas moins hybride, avec ses longues et sinueuses phrases qui se rapprochent du rythme et de la syntaxe de la conversation. La narration elle-même, par son seul usage des temps, dévoile sa construction composite, qui tient à la fois du récit fictif et de l'autobiographie: par moments, on trouve un « je » énonciatif au présent de l'indicatif qui, comme Benveniste l'a expliqué dans Problèmes de linguistique générale (1976), est un temps appartenant au domaine du discours, représentant le factuel, le «réel », et excluant de ce fait la fiction. Les actions représentées au simple past (mais depuis la perspective du présent, celle du discours) correspondent, en français, au passé composé, un temps qui exclut traditionnellement la fiction. Pourtant, lorsque l'auteure-narratrice raconte depuis la perspective d'un passé presque mythique, le choix du passé simple semble naturel, ce temps étant, comme Käte Hamburger (1986) l'a noté après Benveniste, un temps connotant le mode fictif. Ainsi, les deux registres du passé en français, celui du discours et celui du récit, ont été utilisés dans la traduction pour rendre les deux univers temporels du genre composite de The Perfection of the Morning.

\section{La traduction "performative » et ses motifs cachés, ou comment s'approprier un territoire par les mots}

On a vu avec Austin et sa théorie des actes de langage que le fait de nommer une réalité peut la créer, la " performer » ${ }^{4}$. Jonathan Culler, en résumant la question de l'opposition entre les actes constatifs, qui constatent, décrivent une réalité, et les actes performatifs, qui accomplissent, créent l'acte qu'ils nomment, montre comment ces catégories se sont avérées pertinentes pour caractériser le discours littéraire, qui crée tout autant qu'il décrit. Comme le performatif, le littéraire n'est pas directement référentiel, pas plus qu'il n'est vrai ou faux (Culler 1997 : 96-97). Par ailleurs,

\footnotetext{
${ }^{4}$ Voir à ce sujet l'article « Acte de langage » de Catherine Kerbrat-Orecchioni, dans le Dictionnaire d'analyse $d u$ discours préparé. Elle y résume l'hypothèse de départ d'Austin dans Quand dire c'est faire (1970): «'Dire', c'est sans doute transmettre à autrui certaines informations sur l'objet dont on parle, mais c'est aussi 'faire', c'est-à-dire tenter d'agir sur son interlocuteur, voire sur le monde environnant. Au lieu d'opposer comme on le fait souvent la parole à l'action, il convient de considérer que la parole elle-même est une forme et un moyen d'action. À la source de la théorie austinienne, il y a la découverte de l'existence d'un type particulier d'énoncés, les énoncés performatifs, qui ont la propriété de pouvoir dans certaines conditions accomplir l'acte qu'ils dénomment, c'est-à-dire de 'faire' quelque chose du seul fait de le 'dire'» (pp. 16-17.) Voir par ailleurs Jonathan Culler, Literary Theory. A Very Short Introduction,.
} 
L'énonciation littéraire crée les circonstances auxquelles elle se réfère, sous plusieurs aspects. Premièrement, elle met au monde des personnages et leurs actions, par exemple. [...]. En second lieu, les œuvres littéraires créent des idées, des concepts, qu'elles déploient [...]. Bref, le performatif centralise un usage du langage considéré auparavant comme marginal un usage actif, créateur de mondes, qui ressemble au langage littéraire et qui nous aide à concevoir la littérature comme un acte ou un événement. (Ma traduction de Culler 1997 : 97-98)

$\mathrm{Si}$, pour ces raisons, la littérature peut être considérée comme un acte performatif, la traduction, et a fortiori la traduction littéraire, ne peut qu'être un acte performatif.

En outre, si une œuvre comme celle de Butala «performe » (engendre et répète) (Ibid.) une certaine idée de l'Ouest, la traduction récréera cette réalité dans une autre langue. Le langage performatif, comme Derrida l'a montré, doit pouvoir être tout aussi itératif, pour conserver sa performativité, qu'il doit être inaugural. Or, la traduction, étant un acte relationnel, a toujours à voir avec l'inaugural - le texte littéraire qu'elle traduit - et l'itératif, le texte produit dans la langue d'arrivée. Pourtant ce texte, puisqu'il arrive dans un nouveau contexte et utilise une langue qui ne possède pas les mêmes ressources, comporte une part d'inaugural.

Dans un autre ordre d'idées, je ne doute pas que le pouvoir de nommer sa réalité physique en français puisse alléger quelque peu ce sentiment récurrent de disparition propre aux minorités dont Paré a si éloquemment parlé. Ainsi écritil : «Les sociétés périphériques [...] sont puissamment travaillées par l'angoisse de la mort et du silence ». Dans ces sociétés qui, écrit encore Paré, "rompent toute ligature avec la mémoire» (2004: 10), «le sol identitaire semble se dérober » (idem). Je ne connais pas les raisons pour lesquelles le choix de certains Fransaskois ainsi que de l'éditeur La nouvelle plume s'est arrêté sur cet ouvrage de Butala, mais The Perfection of the Morning porte remarquablement peu sur l'histoire car Butala s'intéresse principalement à la terre telle qu'elle a été depuis les dernières glaciations. Autrement dit, Butala s'intéresse ici à la nature dans ce qu'elle a d'immuable. Lire cette méditation en français ne serait-elle pas une manière, non seulement de nommer cette terre et ce qu'elle suscite, de se l'approprier pour ses langue et culture françaises, comme je l'ai mentionné plus haut, mais également d'imaginer un sol stable, une terre idéale sur laquelle

${ }^{5}$ Dans Théories de la fragilité (1994 : 20-21) et Littératures de l'exiguïté (1992). 
l'histoire s'efface à mesure, une terre ahistorique, pour contrer cette perturbante perception d'un espace mouvant, plus marqué chez les sociétés diasporiques ? Ce désir de traduction ne témoignerait-il pas, pour reprendre les mots de Paré, $\mathrm{d}^{\prime}$ « un rapport problématique à l'espace » $(2004: 68)$ ? Autrement dit, le récit du déménagement de Butala de la ville à une région rurale isolée, du passage de la vie d'universitaire à celle de femme de rancher, avec les limitations qu'elle pouvait apporter, ainsi que la glorification d'un paysage quasi-intact pourraientils fonctionner comme une sorte de métaphore pour ces Fransaskois, un espace symbolique évocateur, avec sa description d'une terre mythique parce qu'inchangée ? Cette plaine mythique fonctionnerait comme la face positive de la monnaie : un déplacement de l'angoisse de cette culture vraiment périphérique vers un élément naturel, concret et stable, la terre. La face négative serait représentée par les conséquences émotives du déplacement : ainsi, la traversée d'un désert intérieur dans The Perfection of the Morning, particulièrement dans ce chapitre qu'elle intitule "Anomie », et qui rend compte du désespoir et du vide d'une personne déplacée d'une culture à une autre. Paré, dans La distance habitée, affirme justement la nécessité $d^{\prime}$ ' un espace discursif qui tienne compte de la décomposition et de l'anomie des cultures décentrées » (2004: 75).

On sait la difficile relation des cultures marginalisées avec la mémoire, qui très souvent est humiliation. Paré affirme d'ailleurs : «Plus que toutes les autres, les cultures diasporales tombent littéralement (s'abolissent!) sous la sanction homogénéisante du présent. Elles sont les premières à renoncer au passé et à se projeter dans un imaginaire de la fragmentation, hors de toute saisie diachronique des événements, hors de tout récit fondateur. Elles se méfient de monuments qui cristalliseraient le manque à soi et la distance qui sont au cœur de leur fonctionnement » $(2004: 89)$.

Justement, l'histoire dans The Perfection of the Morning, lorsqu'elle est abordée, est soit toute récente - elle apparaît dans l'actualité couvrant les années durant lesquelles Butala a écrit cet essai autobiographique -, soit liée à son histoire personnelle - la Déportation des Acadiens, par exemple, qui est esquissée à grands traits parce qu'elle met en scène l'acadianité de sa mère. Butala discute également l'histoire des Premières Nations dans sa région avant l'arrivée de Blancs et leurs premières relations avec ces derniers. On pourrait donc avancer que, vraisemblablement pour les besoins de son essai autobiographique, Butala n'a esquissé l'histoire qu'à grands traits, de manière à se concentrer sur les modes de vie ancestraux que sont ceux du rancher ou ceux des Premières Nations telles qu'elles vivaient il y a un siècle ou deux, parce que ces périodes 
sont perçues comme appartenant à un temps cyclique, comme le retour du matin après la nuit. D'où le titre de l'ouvrage. Butala accordant une grande place à la spiritualité, on peut se demander si cette spiritualité pour les Fransaskois n'est pas une façon d'oblitérer sa réalité de minoritaire. Car en ce qui concerne l'histoire individuelle, la mémoire est faite - je cite Paré--« de ruines et de débris. Ainsi s'érige le sens de la précarité » (2004: 40).

Chez Butala, la précarité de cette mémoire incertaine refait surface ici et là, transparente. Et pourtant ailleurs, Butala enfouit cette incertitude propre à la mémoire sous l'idée qui veut que le moi soit stable, et qu'il s'agit seulement de le découvrir sous les couches de fausseté. De là son emploi abondant du qualificatif « real » : a real man, the real nature, my real self. $\mathrm{Si}$, des descriptions et du récit de Butala, à peu près n'importe quelle lectrice ou lecteur déduira que l'identité est construite, c'est-à-dire qu'elle diffère selon le contexte, Butala semble tout de même insister ailleurs dans son récit pour présenter l'identité comme quelque chose que l'on dé-couvre. Ces «real» qu'elle plante dans son texte comme les bornes du naturel me semblent poser problème en ce que ces qualificatifs réduisent à l'ipséité le mouvant et le fugitif dans une identité, qu'elle soit individuelle ou collective. Peut-être est-ce également cette oscillation entre la conscience de la fragilité de l'identité, de sa mouvance selon les expériences, et l'oubli de cet état, oubli qui se caractérise par un désir de monumentaliser, d'arrêter le fugitif, qui a touché les Fransaskois intéressés à l'œuvre de Butala. Car si, comme le dit Paré, la structure que procure la différence est "une condition absolue d'intelligibilité » (2004: 18), alors on comprend un peu ce qui pouvait attirer les Fransaskois dans The Perfection of the Morning: l'hyperconscience du déracinement, puis son oubli dans une nature et un mode de vie restés inchangés ${ }^{6}$.

\section{Conclusion}

J'ose espérer que la traduction d'une cuvre comme The Perfection of the Morning peut être perçue comme structurante par la communauté de lecteurs fransaskois parce qu'elle constitue le seuil où s'effectue la rencontre du soi et de l'autre dans les collectivités, d'abord par la différence des langues, mais également par ce que le texte original alloue de similarités dans la différence, c'est-à-dire en mettant l'accent sur tout ce qui est menacé.

${ }^{6}$ Ce changement dramatique de cadre de vie et d'état civil - traditionnellement vécu par les femmes (qui prend mari prend pays, dit le proverbe français) - poussera Butala à prendre du recul en écrivant. La différence structurante - quoique douloureuse - se fait jour, qui permet paradoxalement à Butala d'écrire, elle qui avait jusque-là considéré le statut d'artiste comme un idéal irréalisable plutôt que comme une manière d'habiter la distance à long terme. 
La préface à la dixième édition de l'édition originale mentionne le fait que les Butala ont donné au gouvernement 13000 acres de terre vierge pour s'assurer qu'elle reste une terre que les bisons et les autres animaux de cette région comme le renard véloce, la chevêche des terriers, des espèces menacées pourront parcourir librement. The Perfection of the Morning me semble, après relecture, être un plaidoyer pour tout être minorisé ou menacé d'extinction, qu'il s'agisse d'êtres humains définis selon leurs sexes, leur travail, leur habitat ou leur ethnie, ou encore d'animaux et de plantes. D'ailleurs l'omniprésence des Premières Nations, parce qu'elle est reconnue dans sa durable minorisation, devient dans le texte traduit en français une ode à l'endurance qui résonne chez toutes les minorités. Si ma contextualisation de cette drôle d'autobiographie est valable, alors sa traduction, paradoxalement, s'avère peut-être une arme pour atténuer la minorisation. 


\section{BIBLIOGRAPHIE}

BENVENISTE, Émile (1976), Problèmes de linguistique générale, Paris, Gallimard.

BUTALA, Sharon (1994), The Perfection of the Morning, Toronto, Harper Collins.

BUTLER, Judith (1999), Gender Trouble, New York, Routledge.

CLIFFORD, James (1997), Routes. Travel and Translation in the Late Twentieth Century, Cambridge et Londres, Harvard University Press.

CULLER, Jonathan (1997), « Performative Language », Literary Theory. A Very Short Introduction, Oxford et New York, Oxford University Press, 95-109.

CRONIN, Michael (2006), Translation and Identity, Londres et New York, Routledge.

HAMBURGER, Käte (1986), Logique des genres littéraires (traduit de l'allemand par Pierre Cadiot; préface de Gérard Genette), Paris, Seuil.

KERBRAT-ORECCHIONI, Catherine (2002), « Acte de langage », dans P. Charaudeau et D. Maingueneau (dirs.), Dictionnaire d'analyse $d u$ discours, Paris, Seuil, 16-19.

PARÉ, François (1992), Les littératures de l'exiguïté, Ottawa, Le Nordir.

PARÉ, François (2004), La distance habitée, Ottawa, Le Nordir. 